(D) Check for updates

Cite this: Food Funct., 2021, 12, 8425

\title{
Development of a separated-dough method and flour/starch replacement in gluten free crackers by cellulose and fibrillated cellulose
}

\author{
Yi Ren, (D) *a Gleb E. Yakubov, ${ }^{a}$ Bruce R. Linter ${ }^{b}$ and Tim J. Foster ${ }^{a}$
}

Two strategies were combined and applied in this study to achieve a desired structure and texture of gluten free crackers and to reduce the calorie content. The first strategy is increasing structural heterogeneity of crackers and doughs and a separated-dough method was developed. A butter dough and a water dough were prepared separately and mixed together and the influence of mixing time was investigated. In the second strategy, which is the incorporation of a structuring material, powdered cellulose and fibrillated cellulose were incorporated in formulation to replace flour and pregelatinised starch with enhanced health benefits of low calorie and high fibre. Powdered cellulose played the role of the skeleton

Received 30th April 2021, Accepted 4th August 2021 DOI: $10.1039 / \mathrm{d} 1 \mathrm{fo} 01368 \mathrm{~h}$ rsc.li/food-function of the gluten free crackers. A laminar structure was observed in crackers when powdered cellulose was initially added to the butter dough. The crackers exhibit high thickness, hardness and fracturability and sharp sound emission which are typically observed in wheat crackers. Pregelatinised starch can be replaced by fibrillated cellulose at a lower addition level.

\section{Introduction}

The gluten free market has been growing to meet the requirement of a gluten-free diet which is the only treatment for coeliac disease. Gluten free crackers have been produced based on or by incorporating buckwheat flour, hemp flour, green tea leaves, carob's germ and seed peel, apple pomace, cactus mucilage or cladode flour with enhanced nutritional qualities including protein content, mineral content, dietary fibre content, antioxidant activity and radical scavenging activity. ${ }^{1-4}$ Nammakuna et al. ${ }^{5}$ evaluated the influence of protein isolates and hydrocolloids on the physicochemical properties of gluten free doughs and crackers and they found that the addition of protein isolates and hydrocolloids allowed the doughs to form a structure with fewer but larger gas cells which is closer to wheat crackers. Hydrocolloids assist the formation of a continuous matrix in gluten free doughs. ${ }^{5}$

In our previous study, psyllium seed husk powder (PSY) and pregelatinised starch (PGS) are found to hold flour particles together and form cohesive doughs. Upon baking, PSY/PGScontaining gluten free crackers exhibit texture properties com-

\footnotetext{
${ }^{a}$ Division of Food, Nutrition and Dietetics, School of Biosciences, University of Nottingham, Sutton Bonington Campus, Loughborough, LE12 5RD, UK. E-mail:yi.ren@nottingham.ac.uk, Gleb.Yakubov@nottingham.ac.uk, Tim.Foster@nottingham.ac.uk

${ }^{b}$ PepsiCo International Ltd, 4 Leycroft Rd, Leicester, LE4 1ET, UK. E-mail: Bruce.Linter@pepsico.com
}

parable to wheat crackers, as determined by texture and sound emission analysis. ${ }^{6}$ However, a laminar or plate-like structure, which is observed in wheat crackers, was absent. ${ }^{6}$ In order to improve the product quality of PSY/PGS-containing crackers and mimic their wheat-based counterparts more closely, this study proposes and combines two strategies that target the achievement of the desired structure of low moisture snacks. The first strategy is increasing structural heterogeneity during processing. To increase the structural heterogeneity, we developed a separated-dough method in this study.

The second strategy is incorporating structuring material. Cellulose fibre can be added as a structured filler material and can be used to replace flour in order to reduce the calorie content. Cellulose is one of the most abundant polysaccharides and widely found in plants as the primary structuring component of the cell walls. Cellulose chains are stabilised by hydrogen bonds and assembled into larger cellulose fibres. ${ }^{7}$ Unlike other hydrocolloids and dietary fibres, cellulose is insoluble and does not bind a large amount of water due to the extensive existence of intra- and inter-molecular hydrogen bonds. Therefore, it can be considered as a relatively inert filler in terms of water-binding capacity. The advantage is that cellulose can be added into formulation at a larger amount and achieve a significant reduction in the calorie content of foods.

Mechanical and chemical treatments on cellulose have also been widely investigated. Cellulose fibrillation by high-speed shearing or pressure causes delamination and unwinding of 
the high-level cellulose structure and generates highly entangled long fibrils. Microfibrillated cellulose is widely used as thickeners, compounds carriers and suspension stabilisers in food, pharmaceutical and cosmetic products. ${ }^{8,9}$ We previously fibrillated cellulose from wood pulp using a colloid mill and obtained a relatively stable suspension with increased water retention ability. ${ }^{10}$ Mechanical fibrillation of cellulose increases the exposure of hydroxyl groups on the surface of cellulose and the interaction with water. ${ }^{10}$ It is also noticed that the fibrillating process significantly increase the pasting viscosity of cellulose-starch blends (Unpublished results). Therefore, compared to native cellulose, fibrillated cellulose becomes similar to gelatinised starch in terms of interaction with water and increase in viscosity.

Therefore, based on the separated-dough method, powdered cellulose (NC) with long fibres and high bulk volume was added into the formulation as a skeleton of crackers and replace flour for lower calorie content and higher fibre content. Additionally, fibrillated cellulose (FC) was added to replace pregelatinised starch in the formula.

\section{Materials and methods}

\section{Materials}

Rice flour with moisture content, protein content, ash content, lipid content, and amylose content of $11.08 \%, 7.23 \%, 0.42 \%$, $2.8 \%$ and $28.79 \%{ }^{11}$ was purchased from Doves Farm. Psyllium seed husk powder (P95, Vitacel $®$ ) was kindly donated by the J. Rettenmaier \& Söhne Group (JRS, Rosenberg, Germany). Pregelatinised waxy maize starch (Merigel $\left.{ }^{\circledR}\right)$ was supplied by Tate \& Lyle (UK). Powdered cellulose (Solka floc 900FCC) was supplied by International Fiber Corporation (US). Solka floc 900FCC has high fibre length $(120 \mu \mathrm{m}$ on average) and high bulk volume $\left(5.2-6.2 \mathrm{~mL} \mathrm{~g}^{-1}\right) .{ }^{12}$ Other essential ingredients included gluten Free Baking Powder (Dr Oetker), caster sugar, salt, unsalted butter and sunflower oil. Fluorescein isothiocyanate (FITC, Acros Organics, New Jersey, US), Nile red (Sigma Aldrich) and Calcofluor white stain $\left(1 \mathrm{~g} \mathrm{~L}^{-1}\right.$, Sigma Aldrich, UK) were of analytical grade.

\section{Cellulose fibrillation}

Fibrillated cellulose (FC) were prepared as described in Ren, et $a l .{ }^{10}$ and Ren, et al. ${ }^{11}$ More specifically, five grams of powdered cellulose (NC) was dispersed in $500 \mathrm{ml}$ of water and fibrillated using a colloid mill (Winkworth, Basingstoke, UK) for $30 \mathrm{~min}$. The resulting suspension was centrifuged $(4000 \mathrm{~g}$, $4^{\circ} \mathrm{C}$ ) for $15 \mathrm{~min}$. The sediment was collected and the concentration of FC for each batch was measured by drying at $105{ }^{\circ} \mathrm{C}$. The concentrated FC paste was stored at $4{ }^{\circ} \mathrm{C}$.

\section{Formulation and cracker preparation}

The formulation of gluten free crackers is shown in Table 1. Butter doughs and water doughs were mixed separately in the separated-dough method. To prepare the butter dough of F0 (B-NC0), all dry ingredients were mixed thoroughly and mixed with diced butter in a Kenwood (UK) stand mixer using a Chef $\mathrm{K}$ beater. Water and oil were then added and mixed at speed 2 for $1 \mathrm{~min}$ and speed 4 for $2 \mathrm{~min}$. The butter dough was stored at $5{ }^{\circ} \mathrm{C}$ for $20 \mathrm{~min}$. To prepare the water dough of F0 (W-NC0), PSY was heated in $21.25 \mathrm{~g}$ of water and cooled down to room temperature. The heated PSY suspension was mixed with all other ingredients at speed 2 for $2 \mathrm{~min}$ and speed 4 for $1 \mathrm{~min}$. The water dough rested at room temperature for $10 \mathrm{~min}$. To make the final mixed dough of F0, water dough was placed in the middle of two portions of butter dough and mixed using the $\mathrm{K}$ beater by switching between the minimum speed and speed 2 for every $10 \mathrm{~s}$. The total mixing time was $40 \mathrm{~s}$ ( 2 cycles of mixing), $60 \mathrm{~s}$ (3 cycles of mixing) or $120 \mathrm{~s}$ (6 cycles of mixing).

For the cellulose enriched doughs (F1 to F6), NC was mixed with other dry ingredients and FC was diluted to the required concentrations calculated from Table 1. PSY was dispersed and heated in the FC suspensions and the mixtures were added to form the water doughs. The total mixing time when combining butter and water doughs was $60 \mathrm{~s}$.

All mixed doughs were placed in a fridge for $10 \mathrm{~min}$, laminated by folding to 16 layers in total, and sheeted to $1.6 \mathrm{~mm}$. The dough sheets were transferred onto baking parchment and cut into $36 \times 36 \mathrm{~mm}$ squares. The crackers were initailly baked at $250{ }^{\circ} \mathrm{C}$ for $6 \mathrm{~min}$ and then at $105^{\circ} \mathrm{C}$ for $10 \mathrm{~min}$ in a fan oven (Hotpoint, UK). The crackers were equilibrated at room temperature and stored in polyethylene bags. The fibre (NC, FC and PSY) content of the crackers was calculated based on the total dry weight in each formulation and listed in Table 1. Based on the rough estimation, F1, F2, F3 and F6 contain fibres higher than $6 \mathrm{~g}$ per $100 \mathrm{~g}$ which, according to Regulation (EC) No. 1924/2006, are expected to be claimed as 'high fibre' products.

\section{Time-domain NMR measurement}

The water and lipid distribution in butter doughs, water doughs and mixed doughs were probed using an R4 Benchtop NMR System (Advanced Magnetic Resonance Ltd, Abingdon, U.K.) at $25{ }^{\circ} \mathrm{C}$. The proton relaxation measurements were performed applying the Carr-Purcell-Meiboom-Gill (CPMG) sequences. ${ }^{13}$ The initial $90^{\circ}$ pulse was approximately $2.8 \mu \mathrm{s}$ and the signals were recorded $5 \mu$ s (dead time) after the $90^{\circ}$ pulse. The TAU was $256 \mu$ s between the following $2048180^{\circ}$ pulses. The signal was obtained averaging 32 scans for each sample with $10 \mathrm{~s}$ relaxing time between every two scans.

\section{Dough rheology}

The rheological behaviours of cracker doughs were measured by frequency sweep tests using an MRC 301 rheometer (Anton Paar, Austria) with serrated parallel plate geometry (PP25/P2SN15766, Anton Paar, $2 \mathrm{~mm}$ measuring gap). The water doughs, butter doughs and mixed doughs were prepared as described in section 2.3 without baking powder. Water doughs and butter doughs were directly loaded on the rheometer. The mixed doughs were laminated and sheeted to $2.5 \mathrm{~mm}$ on the bottom plate. The doughs were allowed to resting for $500 \mathrm{~s}$ 
Table 1 Addition levels of ingredients in gluten free cracker formulation

\begin{tabular}{|c|c|c|c|c|c|c|c|}
\hline Butter doughs & F0 & $\mathrm{F} 1$ & $\mathrm{~F} 2$ & F3 & F4 & F5 & F6 \\
\hline Code & B-NCO & B-NC10 & B-NCO & B-NC5 & B-NCO & B-NCO & B-NC10 \\
\hline Water & 3.75 & 3.75 & 3.75 & 3.75 & 3.75 & 3.75 & 3.75 \\
\hline Flour & 20 & 15 & 20 & 17.5 & 20 & 20 & 15 \\
\hline PGS & 2.5 & 2.5 & 2.5 & 2.5 & 2.5 & 2.5 & 2.5 \\
\hline PSY & 0 & 0 & 0 & 0 & 0 & 0 & 0 \\
\hline Butter & 15 & 15 & 15 & 15 & 15 & 15 & 15 \\
\hline Oil & 5 & 5 & 5 & 5 & 5 & 5 & 5 \\
\hline Salt & 0.5 & 0.5 & 0.5 & 0.5 & 0.5 & 0.5 & 0.5 \\
\hline NC & 0 & 5 & 0 & 2.5 & 0 & 0 & 5 \\
\hline FC & 0 & 0 & 0 & 0 & 0 & 0 & 0 \\
\hline
\end{tabular}

Water doughs

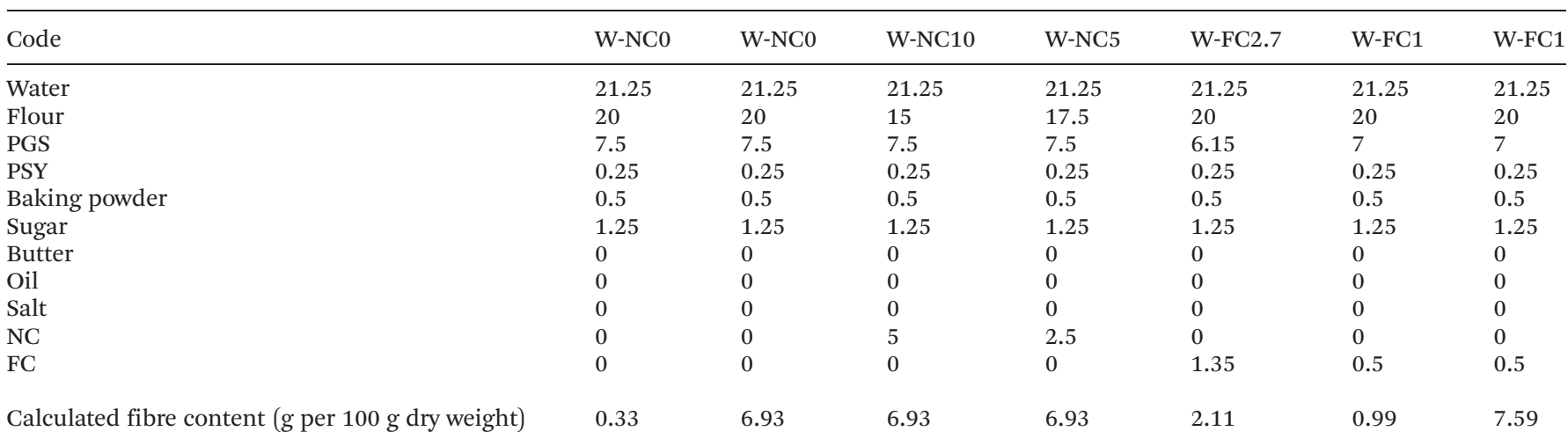

after which the angular frequency decreased logarithmically from 100 to 0.1 rad $\mathrm{s}^{-1}$. A constant shear strain of $0.02 \%$ within the linear viscoelastic region was applied.

The doughs were also evaluated by texture profile analysis (TPA) using a TA-XT plus texture analyser (Stable Micro systems). A $100 \mathrm{~mm}$ plate was attached to a $30 \mathrm{~kg}$ loading cell. A cylinder mode (27.6 $\mathrm{mm}$ in diameter and $18.5 \mathrm{~mm}$ in height) was prepared. All butter and water doughs were filled into the mode to form a cylinder shape. All mixed doughs were laminated, sheeted and stacked layer by layer in the mode. Dough samples were wrapped by cling film and rested for $500 \mathrm{~s}$ at room temperature before measurements. The samples were compressed twice to $65 \%$ of the initial height with a $5 \mathrm{~s}$ resting time between the two compressions to probe the dough properties under large deformation. The testing speed was $1 \mathrm{~mm} \mathrm{~s}^{-1}$. Hardness (first force peak), springiness, cohesiveness, resilience and adhesiveness were calculated from the TPA profiles. The measurements were repeated three times for each dough sample.

\section{Thermal analysis of doughs and crackers}

Differential scanning calorimetry (DSC) analysis on gluten free cracker doughs was performed on a Mettler Toledo DSC 3+ (Mettler-Toledo GmbH Analytical, Switzerland). Cracker doughs were prepared as described in section 2.3. Approximately $10 \mathrm{mg}$ of doughs were hermetically sealed in stainless steel pans. The temperature increased from 5 to
$160{ }^{\circ} \mathrm{C}$ at a heating rate of $5{ }^{\circ} \mathrm{C} \mathrm{min}^{-1}$. The normalised (to flour weights) enthalpy changes $(\Delta H)$ higher than $40^{\circ} \mathrm{C}$ were calculated using STARe software (Mettler-Toledo).

The degree of starch gelatinization of crackers was estimated from DSC measurements. Five milligrams of rice flour or powdered crackers was hydrated overnight in water at a ratio of $1: 3$ in a hermetically sealed aluminium pan. Heat flow was measured from 10 to $100{ }^{\circ} \mathrm{C}$ at a heating rate of $10^{\circ} \mathrm{C} \mathrm{min}^{-1}$. The gelatinisation degree was calculated as described in Sozer et $a .^{14}$ and Ndife et $a .^{15}$ by eqn (1)

$$
\text { Gelatinisation degree }(\%)=\left(1-\frac{\Delta H_{\text {cracker }}}{\Delta H_{\text {flour }}}\right) \times 100
$$

where $\Delta H_{\text {cracker }}$ is gelatinization enthalpy of biscuit samples $\left(\mathrm{J} \mathrm{g}^{-1}\right)$ and $\Delta H_{\text {flour }}$ is gelatinization enthalpy of rice flour $\left(\mathrm{J} \mathrm{g}^{-1}\right)$. An empty pan was used as the reference in all DSC measurements. The measurements were performed in duplicates.

\section{Dough expansion}

The expansion in the thickness of cracker doughs during baking was evaluated using an MRC 301 rheometer equipped with a parallel plate geometry (PP25 SN15766, Anton Paar). The dough samples were prepared and sheeted as described in section 2.3. Wheat cracker dough was also prepared as described in a separate study by our research group ${ }^{6}$ and measured as a positive control. The dough sheet was cut with 
a circular cutter (50 $\mathrm{mm}$ in diameter) and transferred onto the rheometer. The upper plate was lowered at a speed of $10 \mu \mathrm{m}$ $\mathrm{s}^{-1}$ until it touched the dough surface and an increase in normal force was observed. The gap indicated the initial sample thickness. The temperature, which was controlled by a Peltier system (PTD 200) with a temperature-controlled hood, increased from $25{ }^{\circ} \mathrm{C}$ to $180{ }^{\circ} \mathrm{C}$ at a heating rate of $25{ }^{\circ} \mathrm{C}$ $\min ^{-1}$ and kept at $180{ }^{\circ} \mathrm{C}$ for $1 \mathrm{~min}$ to mimic the baking process. The gap was allowed to change during measurements to maintain a constant normal force of $0 \mathrm{~N}$ therefore it represented the thickness of the cracker. The recorded gap was normalised to the initial gap. The measurements were repeated three times for each formulation.

\section{Cracker evaluation}

The thickness of crackers was obtained by averaging the values measured at different positions on the same piece of cracker. Crackers were ground in a coffee grinder and water activity and moisture content were measured.

The textural properties of crackers were evaluated by threepoint bending tests using a texture analyser (TA-XT plus, Stable Micro systems, Surrey, UK). A piece of cracker was placed on two lower supporting points with a distance of $18 \mathrm{~mm}$. The upper probe descended $5 \mathrm{~mm}$ lower than the upper surface of the cracker at a speed of $2 \mathrm{~mm} \mathrm{~s}^{-1}$ and broke the cracker. The force was detected using a $5 \mathrm{~kg}$ loading cell. The sound was recorded simultaneously using an Acoustic Envelope Detector (Stable Micro Systems). Ten to Sixteen crackers were measured for each formulation. Hardness (the maximum force), fracturability (the travelling distance of the probe to the first force peak), maximum sound peak, average peak drops, and number of sound peaks (threshold was 8.5 dB) were recorded.

The hydration of crackers and the development of viscosity during hydration was measured using a Rapid Visco Analyser (RVA) (Newport Scientific Pty. Ltd, Warriewood, New South Wales, Australia) at $37^{\circ} \mathrm{C}$ to assess the mouthfeel of the crackers. Wheat crackers were also prepared as described in Ren, et al. ${ }^{6}$ and measured as a control. The powdered crackers (2.5 g) was added to $15 \mathrm{ml}$ of water in an aluminium canister. The measurements were started immediately and viscosity was measured at a stirring rate of $160 \mathrm{rpm}$ after an initial $2 \mathrm{~s}$ high speed stirring at $960 \mathrm{rpm}$. The tests were performed in triplicates.

\section{Confocal laser scanning microscopy}

The microscopic images of the gluten free cracker doughs were obtained using a Zeiss LSM880 confocal laser-scanning microscope (Carl Zeiss Microscopy GmbH, Jena, Germany) equipped with an EC Plan Neofluar® objective at $20 \times$ magnification. Starch in rice flour and PGS were stained by FITC. Butter and oil were stained by Nile red. PSY, NC, and FC were stained by calcofluor white. Solutions of stains $\left(0.04 \mathrm{~g} \mathrm{~L}^{-1}\right.$ for butter doughs and $0.01 \mathrm{~g} \mathrm{~L}^{-1}$ for water doughs) were added during dough preparation. The excitation wavelengths of FITC, Nile red and Calcofluor white were $488 \mathrm{~nm}, 488 \mathrm{~nm}$ and $405 \mathrm{~nm}$ respectively and the detection wavelength ranges were 508-538 nm, 553-625 $\mathrm{nm}$ and 434-484 nm respectively.

\section{Scanning electron microscopy}

The gluten free crackers were broken from the middle and the exposed surface was evaluated on a scanning electron microscope (JEOL JSM-6060LV, JEOL (UK) Ltd, Welwyn Garden City, UK). A small piece was mounted onto an aluminium stub with the exposed surface facing up and coated with gold using a Leica EM SCD005 Sputter Coater. The argon current rate was $27 \mathrm{~mA}$ and the coating time was $3 \mathrm{~min}$. Multiple images were collected for each sample.

\section{Statistical analysis}

All measurements were repeated at least twice and representative curves and images are shown in figures. Means and standard deviations were calculated which are shown in tables and Fig. 8. One-way analysis of variance (ANOVA) and Tukey's test at a significance level of $p<0.05$ were used for statistical analysis on the experimental data using IBM SPSS statistics version 26 (IBM Corp., Armonk, NY, USA).

\section{Results and discussion}

\section{Development of separated-dough method - the influence of mixing time}

The separated-dough method was developed based on a separate study by our research group ${ }^{6}$ to increase the structural heterogeneity of the cracker doughs. The establishment of the method was initiated with F0 formula without the addition of NC and FC. B-NC0 and W-NC0 dough of F0 (Table 1) were prepared separately and then mixed together for 40 , 60 or $120 \mathrm{~s}$. As expected, the heterogeneity decreased with the increase in the mixing time which can be observed in Fig. 1a, b and $\mathrm{c}$ that the dough sheet mixed for $120 \mathrm{~s}$ had a smoother edge compared to the dough sheet mixed for $60 \mathrm{~s}$ and $40 \mathrm{~s}$.

Water distribution in B-NCO dough, W-NCO dough and mixed doughs of F0 were evaluated using time-domain NMR and the $T 2$ spectra are shown in Fig. 1d. All doughs had a peak or shoulder at approximately $1.5 \mathrm{~ms}$ which is attributed to protons of restrained water in the intragranular space which exchanges with the hydroxyl protons of starch. ${ }^{16-18}$ These intragranular water molecules with short relaxation time are possibly trapped in the crystalline regions of starch granules. ${ }^{18}$ W-NCO dough and the unmixed dough had a $T 2$ peak at about $13 \mathrm{~ms}$ which can be assigned to extragranular water that exists as a thin layer between starch granules which exchange protons with hydroxyl groups of starch and proteins on the granule surface. ${ }^{16,17}$ It could also arise from $\mathrm{CH}$ protons of amylose and amylopectin, as well as water protons in the amorphous regions of starch granules. ${ }^{18,19}$ Apart from W-NC0 dough, all other doughs containing butter had a peak at about $60 \mathrm{~ms}$ and a shoulder or peak at approximate $200 \mathrm{~ms}$ can be sometimes observed. Crackers also showed $T 2$ peaks and shoulders at these relaxation times (data not shown). 

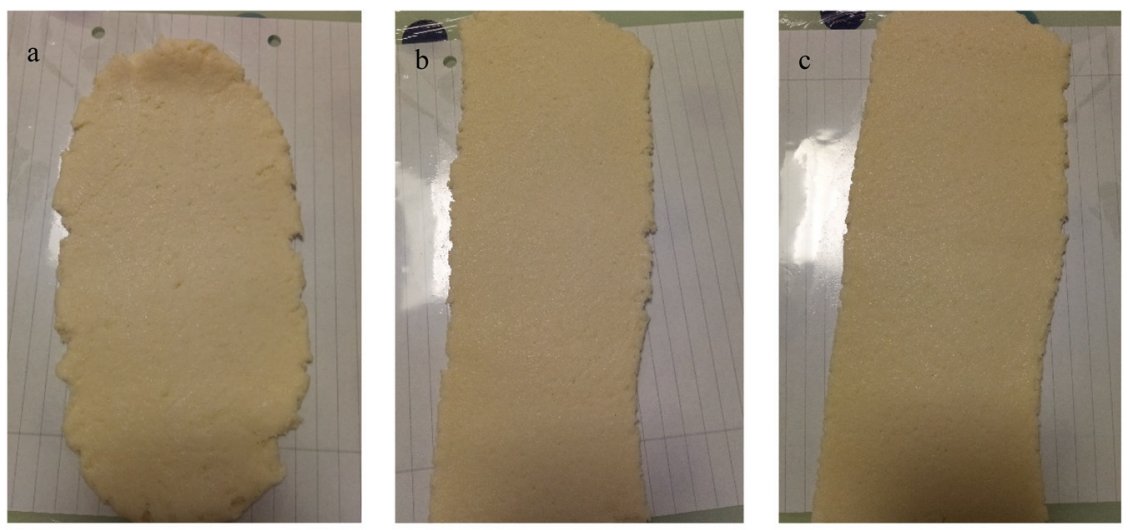

d

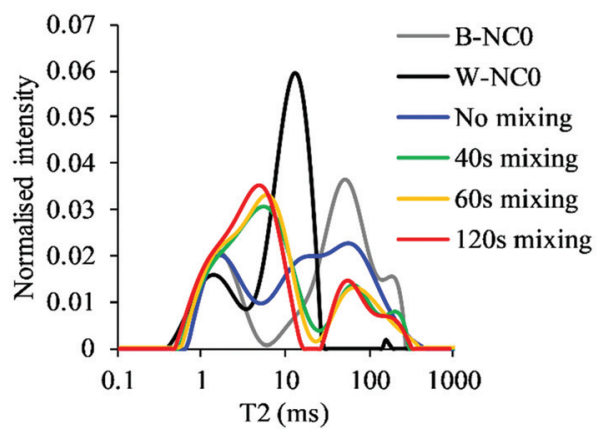

Fig. 1 Gluten free cracker doughs (F0) mixed for $40 \mathrm{~s}(\mathrm{a}), 60 \mathrm{~s}$ (b), and $120 \mathrm{~s}$ (c) after first lamination and distribution proton transverse relaxation times (T2) of B-NCO dough, W-NCO dough and mixed doughs (d).

Considering the low moisture level of the cracker doughs and cracker products, this peak and shoulder are mainly ascribed to the lipid in butter. Two proton populations at similar $T 2$ values were observed for margarine in biscuit doughs. ${ }^{20}$ It is also noticed that the mixed doughs had a $T 2$ peak at about $6 \mathrm{~ms}$ which suggests that the mobility of water was reduced compared to W-NC0 dough and unmixed dough by mixing W-NC0 dough and B-NC0 dough. The width of this peak was narrowed by increasing the mixing time which indicates a reduction of heterogeneity in water distribution.

The influence of mixing time on the hardness and fracturability of crackers was evaluated by three-point bending tests and gelatinisation degrees were measured using DSC which are listed in Table 2. The gelatinisation degree increased with the increase of mixing time. According to $T 2$ spectra which show that heterogeneity of water distribution decreased with the increase in mixing time, it can be speculated that flour in B-NC0 dough, which had lower moisture content than W-NC0 dough, was further hydrated due to the water migration from W-NC0 dough leading to a higher gelatinisation degree. The increase in flour hydration also led to an increase in the water activity and change to a darker colour of the crackers (data not shown). As for cracker hardness, the crackers produced using the separated-dough method all had lower hardness than PSY and PGS crackers (hardness of $801 \mathrm{~g}$ and $667 \mathrm{~g}$ respectively) prepared without the separated-dough method as reported in our separ-
Table 2 Gelatinisation degree of crackers of which the doughs mixed for 40,60 and $120 \mathrm{~s}$

\begin{tabular}{llll}
\hline $\begin{array}{l}\text { Mixing time } \\
(\mathrm{s})\end{array}$ & $\begin{array}{l}\text { Hardness } \\
(\mathrm{g})\end{array}$ & $\begin{array}{l}\text { Fracturability } \\
(\mathrm{mm})\end{array}$ & $\begin{array}{l}\text { Gelatinisation degree } \\
(\%)\end{array}$ \\
\hline 40 & $497 \pm 125^{\mathrm{a}}$ & $0.350 \pm 0.070^{\mathrm{a}}$ & $45.03 \pm 4.19^{\mathrm{a}}$ \\
60 & $525 \pm 97^{\mathrm{a}}$ & $0.349 \pm 0.062^{\mathrm{a}}$ & $63.19 \pm 0.52^{\mathrm{b}}$ \\
120 & $624 \pm 222^{\mathrm{a}}$ & $0.316 \pm 0.060^{\mathrm{a}}$ & $73.44 \pm 5.07^{\mathrm{b}}$
\end{tabular}

Data are shown as mean \pm standard deviation. Means associated with different letters on the same column in each group are significantly different $(p<0.05)$.

ated study. ${ }^{6}$ As for the influence of the mixing time, although the influence was insignificant, a tendency can be observed that hardness increased but fracturability decreased with the increase in mixing time which was associated with a reduction in structural heterogeneity. Because long mixing time led to a reduction in the heterogeneity of structure and water distribution while short mixing time might lead to handling problems and weak structure due to high heterogeneity, an intermediate mixing time, $60 \mathrm{~s}$, was chosen for the following studies.

\section{Mechanical properties of cracker doughs in shear and compression}

The frequency sweeps of B-NC0 dough, W-NC0 dough, and the mixed dough (F0, without cellulose, mixed for $60 \mathrm{~s}$ ) are shown 
a
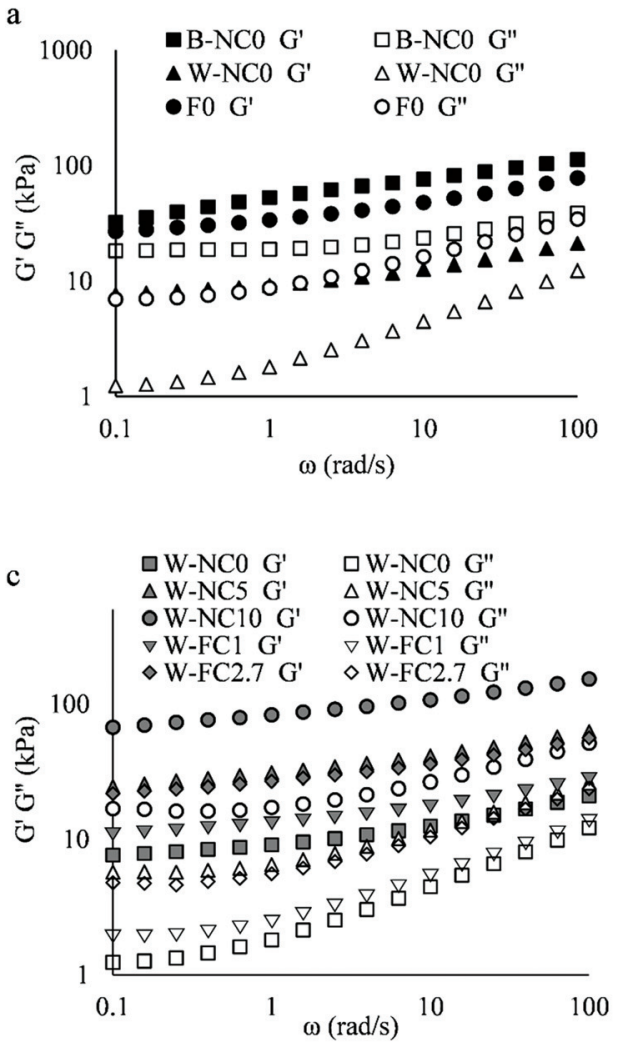

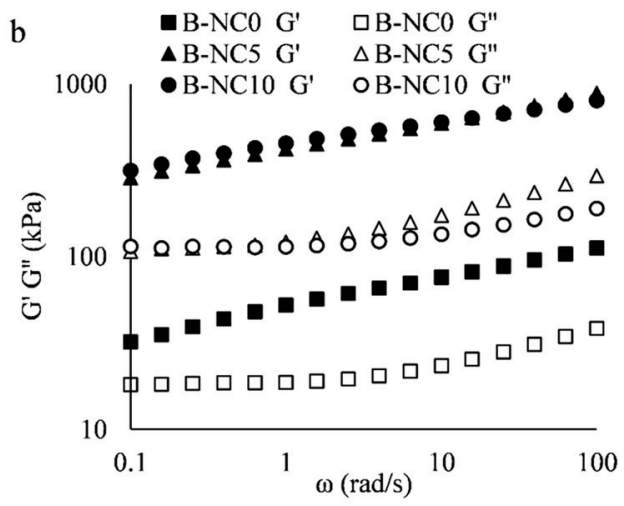

\begin{abstract}
Fig. 2 Frequency sweeps of B-NCO dough, W-NCO dough and mixed dough of F0 mixed for $60 \mathrm{~s}(\mathrm{a})$; frequency sweeps of butter doughs comparing the flour replacement by different amounts of NC (b); and frequency sweeps of water doughs comparing the flour or PGS replacement by different amounts of NC or FC (c).
\end{abstract}

in Fig. 2a. All doughs had solid-like properties with $G^{\prime}$ higher than $G^{\prime \prime}$. The behaviour of $G^{\prime}$ for the B-NC0 dough is characterised by the $G^{\prime}=\omega^{0.22}$ and $\omega^{0.16}$ at frequencies below and above $1 \mathrm{rad} \mathrm{s}^{-1}$, respectively. Overall, the spectrum of B-NC0 dough is similar to what is usually observed for concentrated suspensions. Typically, $G^{\prime}$ of concentrated suspensions exhibits slight dependence on frequency while $G^{\prime \prime}$, which is also weakly dependent on frequency, usually show a shallow minimum at lower frequencies followed by a $G^{\prime \prime}$ peak due to structural relaxation at even lower frequencies before a $G^{\prime}-G^{\prime \prime}$ crossover. ${ }^{21}$ In the case of B-NC0 dough, a $G^{\prime \prime}$ plateau was observed at lower frequencies ( 0.1 to $1 \mathrm{rad} \mathrm{s}^{-1}$ ) instead of a minimum. By contrast, W-NC0 dough had $G^{\prime}$ and $G^{\prime \prime}$ increased at higher angular frequencies while they tended to be frequency-independent at angular frequencies lower than $0.4 \mathrm{rad} \mathrm{s}^{-1}$. This tendency is similar to the spectrum of gluten doughs ${ }^{22}$ which is typically observed for flexible gels of sparsely cross-linked polymers. It can be taken as some evidence for the presence of the weak network formed in W-NC0 dough. The mixed dough (F0) combines the properties of both doughs.

Comparing B-NC0, B-NC5 and B-NC10 (the butter doughs of F0, F3 and F1/F6) as shown in Fig. 2b, the flour reduction by NC significantly increased both moduli by about 7 times. Surprisingly, different concentration of cellulose did not elicit a major influence on the values of $G^{\prime}$ and $G^{\prime \prime}$ of B-NC5 and
B-NC10 under conditions of small amplitude oscillatory shear tests. Nevertheless, the NC addition slightly reduced the frequency dependence of $G^{\prime}$.

Comparing W-NC0, W-NC10, W-NC5, W-FC2.7 and W-FC1 (the water doughs of F0, F2, F3, F4 and F5) as shown in Fig. 2c, the flour or PGS replacement by NC or FC significantly increased $G^{\prime}$ and $G^{\prime \prime}$ and decreased frequency-dependence of $G^{\prime}$ which was similar to the effects of NC on butter doughs. The decreasing effects on the frequency-dependence of $G^{\prime}$ of both butter doughs and water doughs suggest that the addition of NC or FC reinforced the dough structure.

The frequency sweeps of mixed doughs are shown in Fig. 3. Compared to F0, flour replacement by NC (F1, F2 and F3) increased $G^{\prime}$ and $G^{\prime \prime} 2$ to 3 times (Fig. 3a) and PGS replacement by FC (F4 and F5) increased the moduli 1.5 to 1.9 times (Fig. 3b). F2 had slightly lower moduli than F1 and F3. However, the portion of NC added in butter or water dough and the increased addition of FC had a weak influence on shear spectra. Nevertheless, the incorporation of NC and FC together (F6) was found to have a stronger effect than FC addition to the water doughs alone.

The properties of cracker doughs under large deformation in the normal direction were evaluated by TPA tests and the results are shown in Table 3. The increasing effects of the flour reduction by NC and PGS reduction by FC on hardness of 


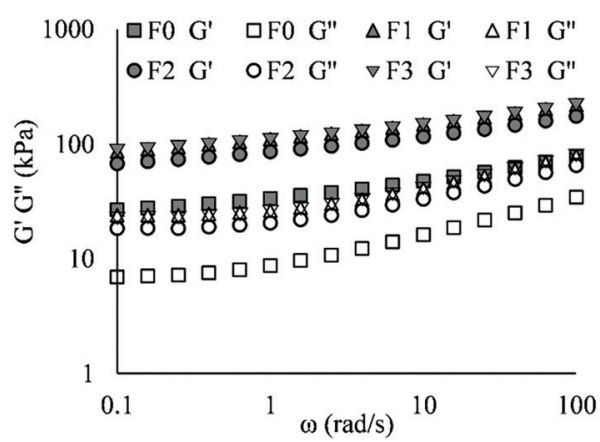

$\mathrm{b}$

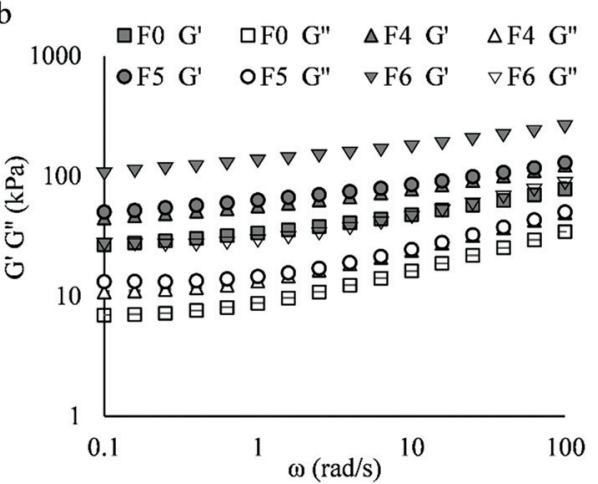

Fig. 3 Frequency sweeps of cracker doughs comparing flour replacement by NC in butter doughs (a) and PGS replacement by FC in water doughs (b).

Table 3 Hardness, springiness, cohesiveness, resilience, adhesiveness, and transition enthalpy $(\Delta H)$ of gluten free cracker doughs

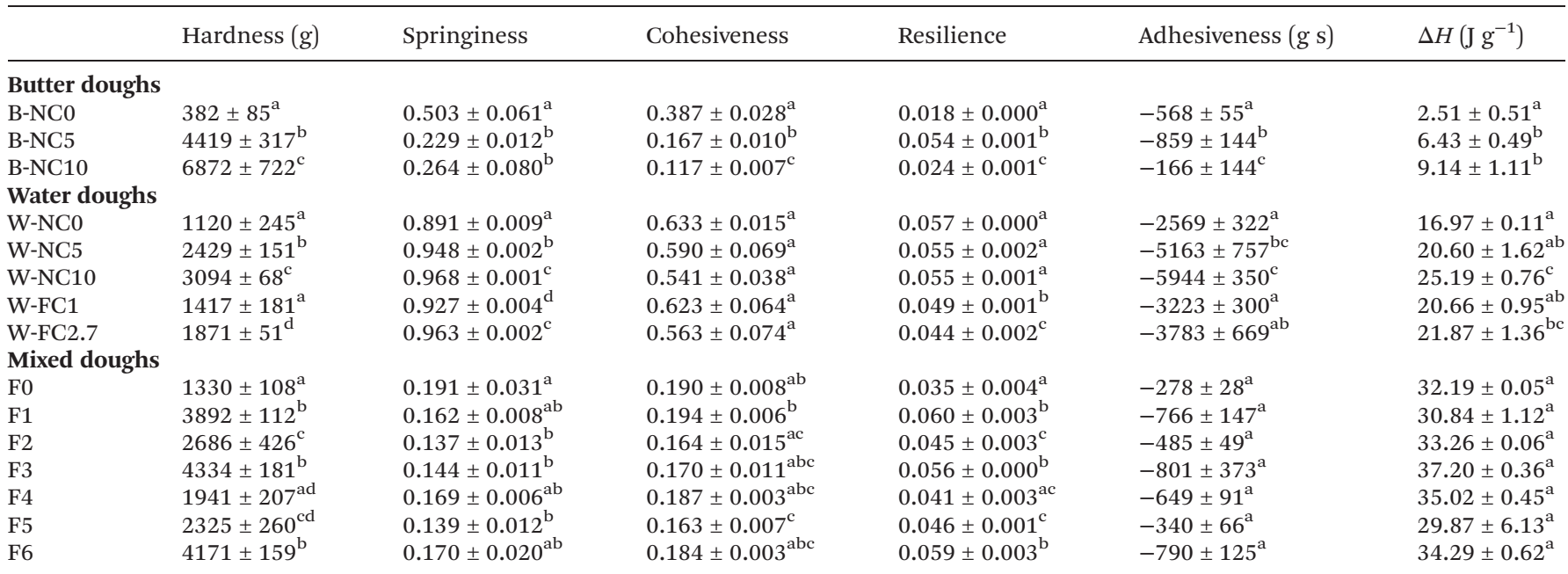

Data are shown as mean \pm standard deviation. Means associated with different letters on the same column in each group are significantly different $(p<0.05)$.

butter and water doughs were generally consistent with their influences on the shear moduli. Hardness kept increasing with the increase of replacement levels which suggests that the large deformation tests under compression could be more sensitive to the additions of NC or FC than small deformation oscillatory tests. The NC and FC reinforced the doughs by the long and entangled fibres and water-binding abilities which increased the resistance to structural destruction under large deformation. However, the addition of NC in butter doughs decreased springiness, cohesiveness and adhesiveness. The low springiness and cohesiveness are due to the plasticising effect of butter/oil and the lack of network forming compounds. As for the water doughs, although flour reduction by NC and PGS reduction by FC slightly increased springiness, it could be due to the high adhesiveness. It was observed that the water dough samples were stuck to the upper plate and pulled up during probe withdrawing. The addition of NC or FC in water doughs tended to increase adhesiveness due to exten- sive interaction between cellulose, starch (from rice flour and PGS) and PSY which strengthened doughs in tensile deformation. It is also noticed that the influence on cohesiveness of water doughs was insignificant and resilience was not influenced by the flour reduction by NC while decreased by the PGS reduction by $\mathrm{FC}$.

Comparing F1, F2 and F3 doughs to F0, the replacement by NC increased hardness. When NC was all added to the water dough, F2 dough was softer than F1 and F3 which is similar to the influences on shear moduli. The increase in hardness was accompanied by a reduction in springiness and cohesiveness and an increase in resilience. Comparing F4 and F5 to F0, the PGS replacement by FC also increased hardness and resilience but decreased springiness and cohesiveness. F6 had similar values to $\mathrm{F} 1$ as both of them contained NC mixed in their butter doughs. Although the difference between adhesiveness of F0 to F6 was insignificant, a tendency can be noticed that the addition of NC or FC increased the adhesiveness. 


\section{Microstructure of cracker doughs}

The microstructure of butter doughs, water doughs and mixed doughs are shown in Fig. 4. In B-NC0 dough, flour particles, which were stained in green/yellow, were dispersed in butter and oil which were stained in red. In B-NC10 dough, flour was partially replaced with NC (blue) which was dispersed in butter and oil the same as flour particles. As for the water doughs, W-NC0 dough was dominantly structured by PGS (green) and PSY (blue). The insert magnifies the structure formed by PGS and rice flour. Some rice starch granules, which was less hydrated and stained due to the competition for water with PGS, can be identified based on their small granule size (about $5 \mu \mathrm{m}$ ) and the polygonal shape. PGS, in the contrast, formed a relatively continuous matrix where rice starch was embedded in. In W-NC10 dough, rice flour was partially replaced by NC and the NC fibres can be observed (blue areas). After fibrillation, FC appears to be a suspension of fibre flocculates. ${ }^{10}$ The FC flocculates or aggregates, which was added to replace PGS, can be observed in W-FC1 dough. Although FC and PSY appeared similar in the overlaid image of W-FC1 dough, FC can be distinguished as fibrous flocculates and PSY are more like particles in the single-channel image (image not shown).

F0 dough was formed by mixing B-NC0 dough and W-NC0 dough. In F0 dough, complexes of flour, PGS and PSY originally constructed in W-NCO dough was surrounded by lipid and some dispersed flour particles from B-NCO dough can also
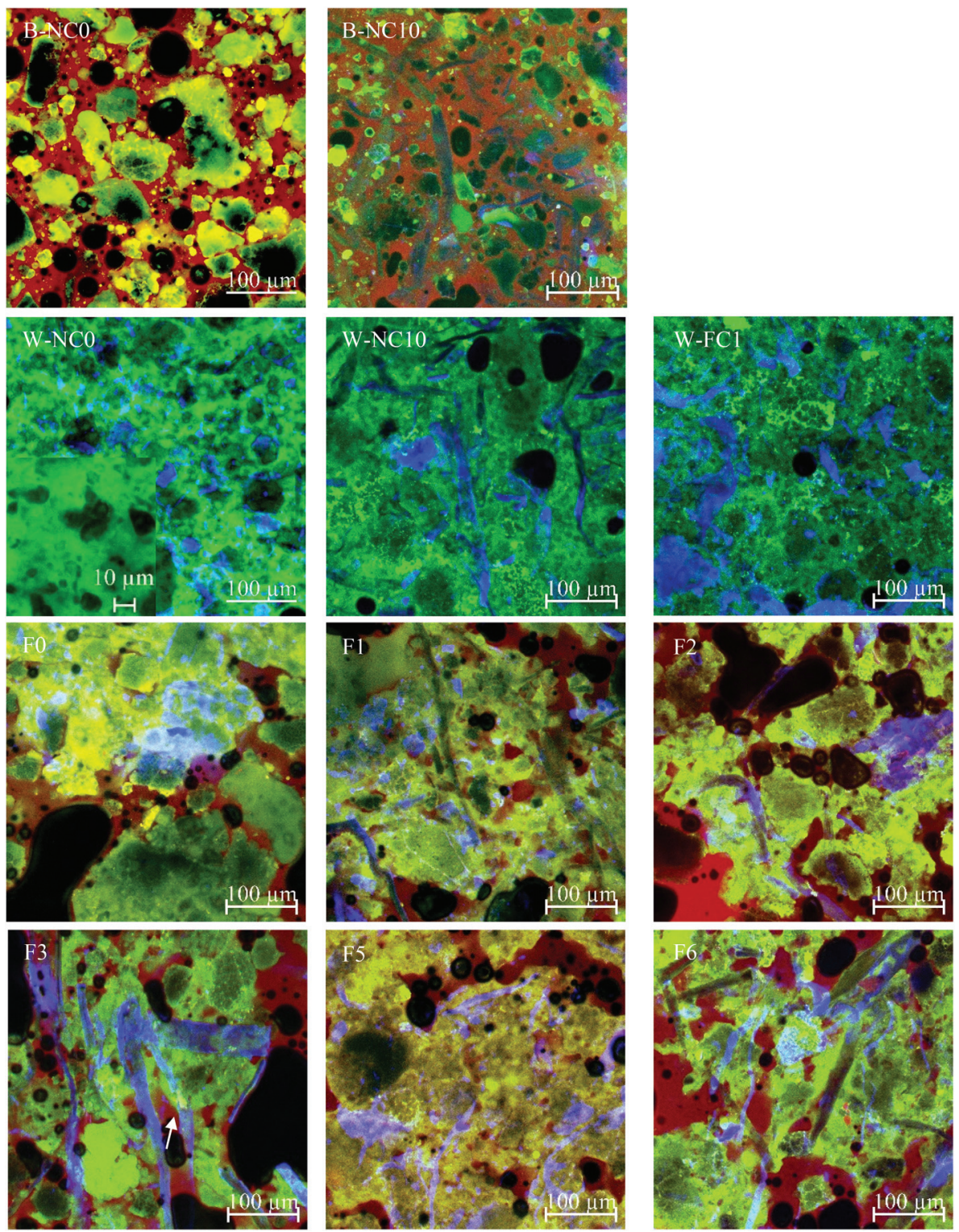

Fig. 4 Confocal laser scanning microscopic images of butter doughs, water doughs and mixed doughs. Starch (both PGS and starch from rice flour) and lipid were stained by FITC and Nile red and shown in green and red respectively. PSY, NC and FC were stained by Calcofluor white and shown in blue. 
be observed. Comparing F1, F2, and F3 doughs, the NC fibre in F1 dough was less stained. It is possible because that NC in F1 was initially added in the butter dough (B-NC10) and the low water addition level of the butter dough limited the hydration and staining of NC fibres. Additionally, the NC fibres were coated with lipid which further limited the hydration and staining even after being mixed with water dough. Nevertheless, there was no significant difference in structure and distribution of ingredients between F1, F2 and F3 doughs which is in agreement with their similar frequency sweep data observed in Fig. 3a. In F5 dough, the complexes constructed by PGS, PSY, and FC surrounded in lipid can be observed and NC in F6 dough can be observed additionally.

\section{Dough expansion during baking}

The expansion of the cracker doughs during baking was monitored by measuring the changes in thickness during heating and the normalised curves are shown in Fig. 5. Wheat crackers and F0 are shown in both Fig. 5a and b to compare with other cracker formulations. Wheat crackers exhibited the highest expansion in thickness during baking. The F0 crackers prepared using the separated-dough method rose to a lower degree. The addition of NC in butter doughs significantly increased the expansion of F1 (Fig. 5a) and F6 (Fig. 5b). It is probably because of the fibrous structure and high bulk volume of NC fibres which were dehydrated and formed the skeleton of crackers. NC in butter dough was coated by lipid which restrained the interactions with water and other hydrophilic materials and allowed the expansion during baking. However, when NC was added to water dough (F2 and F3), the expansion was significantly reduced (Fig. 5a). It is likely due to more extensive interactions with water, hydrated PSY, PGS and flour and a more compact structure was formed which restrained the structural rearrangement and expansion during baking. The weak interaction between cellulose and PSY was also reported in our previous study. ${ }^{10}$ As shown in Fig. 5b, the addition of FC $(0.5 \mathrm{~g})$ replacing PGS in water dough (F5) significantly reduced the expansion compared to F0. However, with further FC addition to $1.35 \mathrm{~g}, \mathrm{~F} 4$ had a similar rising profile to F0.

\section{Thermal properties of cracker doughs and influences on gelatinisation}

The thermal properties of the gluten free cracker doughs were evaluated using DSC and a typical DSC trace of F0 dough is shown in Fig. 6. Two endothermic peaks at 14 and $34{ }^{\circ} \mathrm{C}$ were observed due to the melting of butter. Two melting peaks for butter at the same temperatures were also reported previously because of the multiphasic structure of butter consisting of low melting liquid fractions and high melting solid fractions. ${ }^{23}$ These two peaks might be associated with the two proton populations of butter ascribed to the two $T 2$ peaks as discussed in section 3.1. Rice flour in excess water $(1: 3 \mathrm{w} / \mathrm{w})$ showed a single endothermic peak for starch gelatinisation. However, multiple peaks were observed from 50 to $130{ }^{\circ} \mathrm{C}$ for the cracker doughs due to the low moisture content. Multiple peaks for gelatinisation at low moisture content have also been

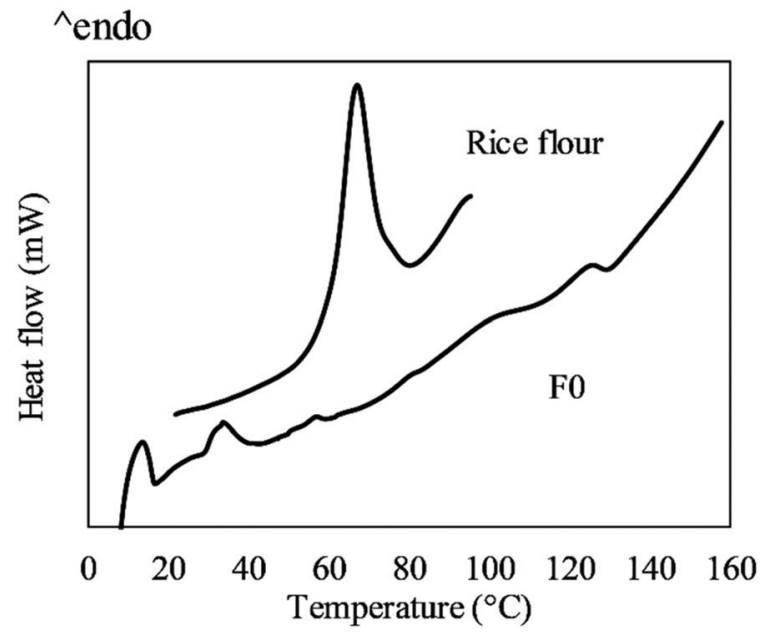

Fig. 6 DSC thermographs of rice flour and FO dough scanned at a heating rate of $5^{\circ} \mathrm{C} \mathrm{min}^{-1}$.
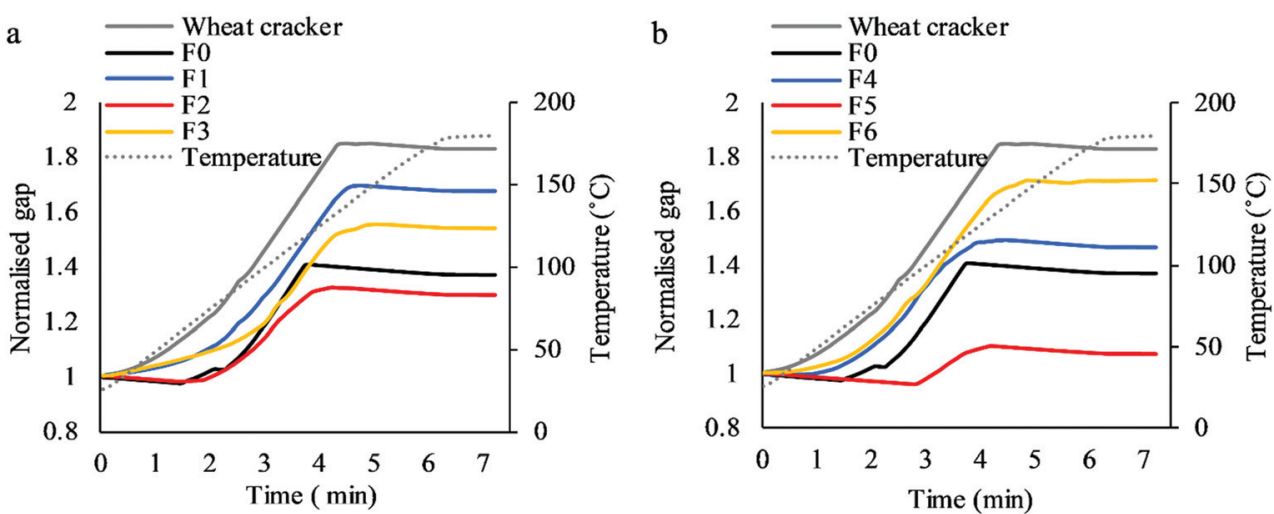

Fig. 5 Increase in thickness detected as gap increase between two plates comparing flour replacement by NC in butter doughs (a) and PGS replacement by FC in water doughs (b). 
reported for wheat, potato and rice starch..$^{23-26}$ The multiple peaks are attributed to multiple thermal events including melting and recrystallization of starch and melting and formation of amylose-lipid complexes. ${ }^{24}$ The total transition enthalpies $(\Delta H)$ of the changes in rice flour and starch were calculated based on the weights of flour and shown in Table 3. Water doughs generally had higher transition enthalpies than butter doughs because of the high water addition level. The flour replacement by NC in butter doughs and flour/PGS replacement by NC/FC in water doughs increased the transition enthalpy. In contrast, reduced gelatinisation enthalpy with the addition of bacterial cellulose to starch has been reported at high water contents with single endothermic peaks observed. ${ }^{27}$ The water content in cracker formulation was much lower while the flour reduction significantly increased the water-to-flour ratio. In addition, PGS, which has high water-binding ability, was replaced by FC. Therefore, flour replacement by NC and PGS replacement by FC allowed more water to be available for starch gelatinisation, which led to the increased transition enthalpy. Additionally, cellulose might interact with starch and stabilise the crystalline regions and increase the energy required for starch gelatinisation and melting. Similar effects have been reported with soluble sugars and hydrocolloids. $^{28-30}$ The influence by flour/PSG reduction on the transition enthalpies of mixed cracker doughs was, however, insignificant except that F3 tended to display a higher value.

\section{Cracker properties}

The dimensions, thickness increases, water activities and moisture contents of the gluten free crackers are shown in Table 4. All crackers had lengths slightly higher than the length of the cracker dough pieces $(36 \mathrm{~mm})$ before baking. Apart from F4, all NC or/and FC added crackers, especially F6, F1 and F3, had comparable increases in thickness to wheat crackers $\left(156 \%\right.$ as reported in our previous study $\left.{ }^{6}\right)$.

F1, F2, F3, F4 and F5 had higher water activities and moisture contents than F0. Additionally, F2 had higher values for these two parameters than F1. It suggests that the flour replacement by NC and PGS replacement by FC reduced the water loss during baking and trapped moistures within the end products which is similar to the incorporation of gums as reported by Kaur et al. ${ }^{31}$

The gelatinisation degrees of gluten free crackers were calculated and shown in Table 4. Similar to the transition enthal- pies of doughs as listed in Table 3, although the difference between the formulations was insignificant, F3 showed the highest gelatinisation degree. However, it seems that the gelatinisation degree and transition enthalpies were not correlated to other cracker quality parameters.

Hydration, dissolution and breakdown of solid compounds and particles during oral processing lead to changes in viscosity and influence oral perception. ${ }^{32}$ The hydration ability of crackers and the viscosity development was monitored using an RVA and the viscosity curves are shown in Fig. 7. The enzymatic hydrolysis of starch and the interactions with other salivary compounds were not of interest in this study. The viscosity in the first $20 \mathrm{~s}$ is not shown due to high noise. As shown in Fig. 7a, the viscosity of wheat crackers increased from $20 \mathrm{cP}$ to $28 \mathrm{cP}$ in the first $100 \mathrm{~s}$ and kept constant in the following $200 \mathrm{~s}$. However, the viscosity of gluten free crackers kept increasing during the $300 \mathrm{~s}$ measurement duration and showed higher values than wheat crackers. The viscosity increase of $\mathrm{F} 0$ started at $13 \mathrm{cP}$ and reached $45 \mathrm{cP}$ at the end of the measurement. F1 and F3 showed similar viscosity profiles that viscosity increased from $20 \mathrm{cP}$ to $45 \mathrm{cP}$. However, F2, where NC was added in water dough, was relatively low in overall viscosity. As shown in Fig. 7b, the PGS reduction by FC (F5 and F4) evidently reduced the overall viscosity which was even lower than wheat cracker during the first $120 \mathrm{~s}$. With the addition of NC in the butter dough, F6 had a higher viscosity profile.

\section{Texture properties and acoustic emission}

Hardness and fracturability of crackers were characterised using three-point bending measurements and the sound released during breakage were recorded which are shown in Table 5. Comparing F1 and F3 to F0, hardness was significantly increased when NC replaced a portion of the flour in butter doughs. Comparing F2 to F0, hardness was also increased when NC was added in water dough; however, it was less efficient than flour replacement in butter doughs (F1 and F3). The PGS replacement by FC in the water doughs of F4 and F5 tended to decrease hardness although the influence was insignificant. Nevertheless, it is noticed that F1 and F6 had similar hardness which suggests that flour replacement by NC in butter dough was predominant in determining the hardness. The influences of the replacement by NC or FC on frac-

Table 4 Dimensions, thickness increases, water activities and moisture contents of the gluten free crackers

\begin{tabular}{|c|c|c|c|c|c|c|}
\hline & Length (mm) & Thickness (mm) & Thickness increase (\%) & Water activity & Moisture content (\%) & Gelatinisation degree (\%) \\
\hline F0 & $36.84 \pm 0.51^{\mathrm{a}}$ & $3.28 \pm 0.12^{\mathrm{ab}}$ & $105 \pm 8^{\mathrm{ab}}$ & $0.27 \pm 0.02^{\mathrm{a}}$ & 3.41 & $56.96 \pm 8.43^{\mathrm{a}}$ \\
\hline F1 & $36.64 \pm 0.73^{a}$ & $3.63 \pm 0.38^{b c}$ & $127 \pm 24^{b c}$ & $0.33 \pm 0.01^{\mathrm{b}}$ & 5.01 & $62.49 \pm 5.37^{\mathrm{a}}$ \\
\hline F2 & $37.14 \pm 0.75^{\mathrm{a}}$ & $3.46 \pm 0.27^{\mathrm{abc}}$ & $116 \pm 17^{\mathrm{abc}}$ & $0.45 \pm 0.01^{c}$ & 6.31 & $67.48 \pm 5.67^{\mathrm{a}}$ \\
\hline F3 & $36.79 \pm 0.59^{a}$ & $3.61 \pm 0.17^{b c}$ & $125 \pm 10^{\mathrm{bc}}$ & $0.33 \pm 0.01^{\mathrm{b}}$ & 4.80 & $70.62 \pm 5.83^{\mathrm{a}}$ \\
\hline F4 & $36.71 \pm 0.97^{\mathrm{a}}$ & $3.01 \pm 0.06^{\mathrm{a}}$ & $88 \pm 4^{\mathrm{a}}$ & $0.40 \pm 0.01^{\mathrm{d}}$ & 5.67 & $66.99 \pm 2.07^{\mathrm{a}}$ \\
\hline F5 & $36.91 \pm 0.73^{\mathrm{a}}$ & $3.50 \pm 0.13^{a b c}$ & $119 \pm 8^{a b c}$ & $0.19 \pm 0.00^{\mathrm{e}}$ & 4.18 & $62.17 \pm 0.00^{\mathrm{a}}$ \\
\hline F6 & $36.88 \pm 0.27^{\mathrm{a}}$ & $4.01 \pm 0.40^{\mathrm{c}}$ & $150 \pm 25^{\mathrm{c}}$ & $0.19 \pm 0.01^{\mathrm{e}}$ & 3.86 & $55.23 \pm 3.22^{\mathrm{a}}$ \\
\hline
\end{tabular}

Data are shown as mean \pm standard deviation. Means associated with different letters on the same column are significantly different $(p<0.05)$. 

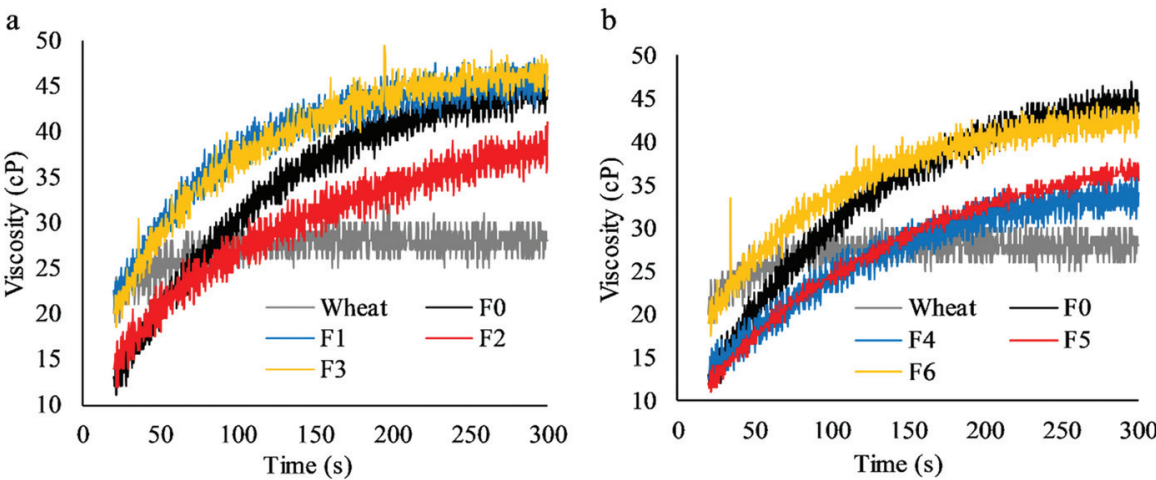

Fig. 7 The RVA viscosity of powdered wheat crackers and gluten free crackers (F0, F1, F2, F3 (a); F0, F4, F5, F6 (b)) during hydration.

Table 5 Texture properties and acoustic emission of gluten free crackers

\begin{tabular}{llllll}
\hline & Hardness $(\mathrm{g})$ & Fracturability $(\mathrm{mm})$ & Maximum sound peak $(\mathrm{dB}(\mathrm{SPL}))$ & Averages of sound peaks $(\mathrm{dB}(\mathrm{SPL}))$ & Number of sound peaks \\
\hline F0 & $525 \pm 97^{\mathrm{a}}$ & $0.349 \pm 0.062^{\mathrm{a}}$ & $86.19 \pm 2.43^{\mathrm{abc}}$ & $64.21 \pm 1.01^{\mathrm{a}}$ & $45 \pm 7^{\mathrm{a}}$ \\
F1 & $2974 \pm 394^{\mathrm{b}}$ & $0.868 \pm 0.126^{\mathrm{b}}$ & $88.46 \pm 2.67^{\mathrm{a}}$ & $66.03 \pm 1.31^{\mathrm{b}}$ & $33 \pm 17^{\mathrm{b}}$ \\
F2 & $1454 \pm 182^{\mathrm{c}}$ & $0.682 \pm 0.081^{\mathrm{c}}$ & $83.98 \pm 3.32^{\mathrm{b}}$ & $66.94 \pm 0.96^{\mathrm{b}}$ & $28 \pm 5^{\mathrm{bc}}$ \\
F3 & $2330 \pm 520^{\mathrm{d}}$ & $0.797 \pm 0.104^{\mathrm{bc}}$ & $86.52 \pm 3.81^{\mathrm{abc}}$ & $66.67 \pm 1.14^{\mathrm{b}}$ & $18 \pm 7^{\mathrm{c}}$ \\
F4 & $363 \pm 36^{\mathrm{a}}$ & $0.463 \pm 0.106^{\mathrm{a}}$ & $79.19 \pm 2.57^{\mathrm{d}}$ & $66.82 \pm 0.73^{\mathrm{b}}$ & $20 \pm 6^{\mathrm{c}}$ \\
F5 & $592 \pm 69^{\mathrm{a}}$ & $0.370 \pm 0.108^{\mathrm{a}}$ & $85.01 \pm 2.10^{\mathrm{bc}}$ & $60.48 \pm 1.39^{\mathrm{a}}$ & $61 \pm 14^{\mathrm{d}}$ \\
F6 & $3172 \pm 608^{\mathrm{b}}$ & $1.031 \pm 0.193^{\mathrm{d}}$ & $87.29 \pm 2.33^{\mathrm{ac}}$ & $32 \pm 7^{\mathrm{b}}$
\end{tabular}

Data are shown as mean \pm standard deviation. Means associated with different letters on the same column are significantly different $(p<0.05)$.

turability were similar to the influences on hardness and F6 had a remarkably higher value.

Sound emission is a critical parameter in defining the perception of crispness. ${ }^{33}$ The maximum sound peak, average peak drop and number of sound peaks are shown in Table 5 to illustrate the sound emission during three-point bending tests. F1, F3 and F6, in which NC was added in the butter doughs, tended to have a higher maximum sound peak than F0 although the difference was insignificant. However, flour or PGS replacement by NC or FC in water doughs (F2, F4, and F5) tended to decrease the maximum sound peak. The observation is coincident with the influence on hardness. The average of sound peaks is another parameter that was found to be corre- lated to the instrumental evaluation of crispness. $^{34-36}$ It is noticed that the flour and PGS replacement by NC and FC (F1, F2, F3 and F4) slightly increased the averages of sound peaks and decreased number of sound peaks. Each occurrence of sound peaks is associated with a structural destruction event. ${ }^{37,38} \mathrm{~F}$, which had the highest number of sound peaks, possibly strengthened the local structure of crackers and some small destruction events led to more sound peaks to be detected. F6 had a lower average and number of sound peaks which suggests a lower overall sound emission. Small sound peaks (lower than $8.5 \mathrm{~dB}$ ) corresponding to minor structure destruction events were not analysed. The highest ten acoustic peaks were arranged from high to low and shown in Fig. 8. It

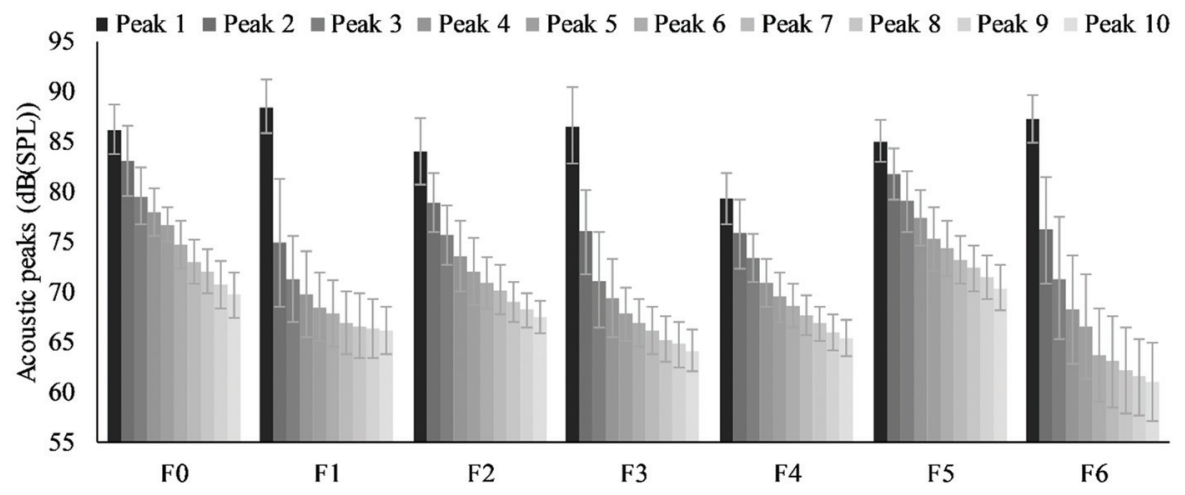

Fig. 8 The highest ten acoustic peaks captured during three-point bending tests on gluten free crackers. The acoustic peaks from peak 1 to peak 10 are arranged from the highest to the lower values from left to right. 
can be seen that the difference between the highest peak and the second highest peak of F1, F3 and F6 was bigger than other crackers which suggest sharper breakage and release of the acoustic energy. This observation is similar to wheat crackers as reported in our previous study. ${ }^{6}$

\section{Microstructure of crackers}

The microstructures of gluten free crackers are shown in Fig. 9. As shown in Fig. 9a, F0 had a relatively heterogeneous structure with voids and cavities. The structural heterogeneity provides multiple breaks during biting which is critical to the texture of crackers. Fig. 9b shows a more detailed structure that the insoluble components including flour particles, rice starch granules, granule fragments of PGS, and the insoluble core of PSY were embedded in a relatively continuous matrix composed of soluble and hydratable components, butter and oil. Rice flour particles can be identified according to the sizes (90\% of the particles are smaller than $200 \mu \mathrm{m}$ with a mean size of $118 \mu \mathrm{m}$, data not shown). As illustrated in Fig. 9c, F1 crackers had a laminar structure which is typically observed in wheat crackers. $^{39}$ The NC fibres can be observed in Fig. 9d embedded in or pointing out the matrix. The NC fibres formed the skeleton for the crackers and, therefore, the structure can be described as fibrous instead of plate-like which was

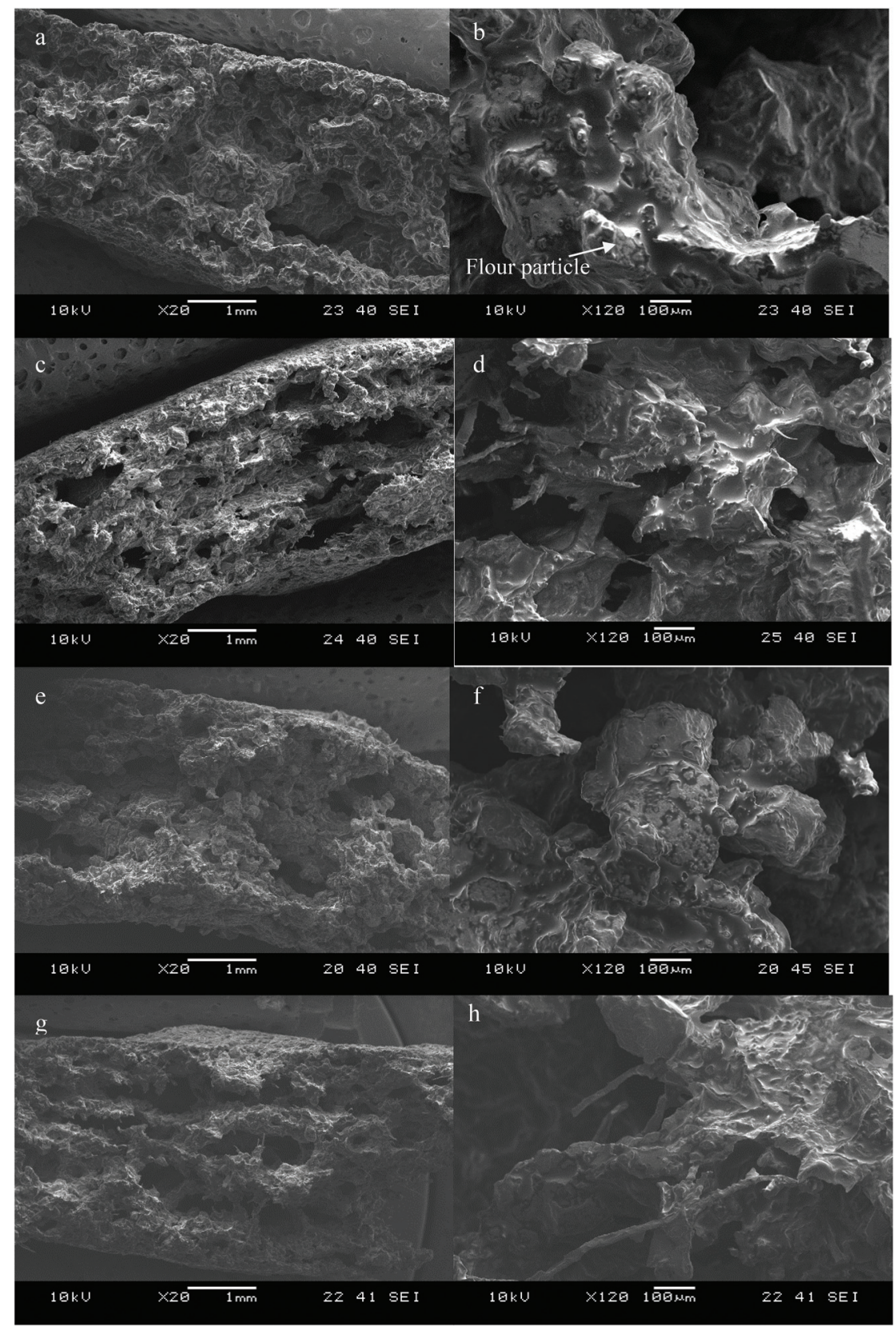

Fig. 9 Scanning electron microscopic images of F0 (a, b), F1 (c, d, o), F2 (e, f), F3 (g, h), F4 (i, j), F5 (k, l), and F6 (m, n) crackers. 


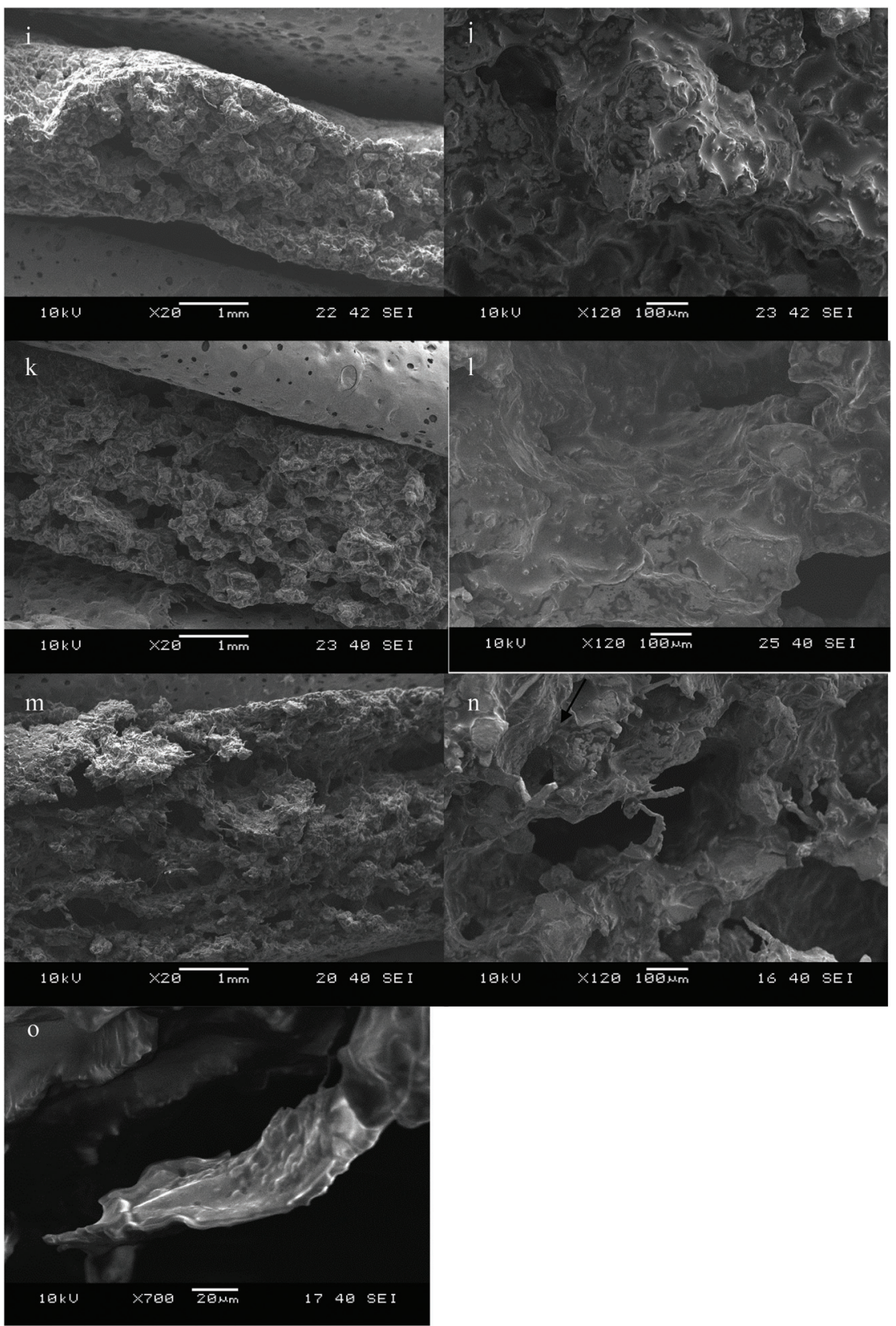

Fig. 9 (Contd).

observed in wheat crackers. ${ }^{6}$ It is likely that NC fibres were aligned during dough sheeting and formed layers. Voids and cavities were formed between these layers as the result of the leavening effect of baking powder and loss of moisture during baking and NC fibres reinforced these layers and contributed to a laminar structure and higher hardness. Additionally, Fig. 9o illustrated that the FC fibres pointing out the matrix were coated by PGS and rice flour which further strengthened these individual fibres and contributed to the texture and sound emission of crackers. F3 (Fig. 9g and h) and F6 (Fig. 9m and $\mathrm{n}$ ) both had FC added in butter doughs and had a similar structure to F1. However, when $\mathrm{NC}$ was added into water dough, F2 crackers did not display a laminar structure although voids and cavities are observed (Fig. 9e). The NC fibres in F2 crackers were mainly embedded in the matrix and not easily observed (Fig. 9f). In this case, NC did not participate in the formation of the laminar structure of the crackers. F5 crackers, when a smaller amount of PGS in water dough was replaced by FC, had a similar structure to F0 crackers. However, with a higher addition level of FC, F4 crackers were thinner and less even with fewer voids and cavities than F0 crackers. The compact structure of F4 crackers also led to the high moisture content and water activity as shown in Table 4 . 


\section{Conclusion}

In this study, two strategies were combined and applied to improve the textural qualities of gluten free crackers prepared based on rice flour with the additions of PSY and PGS. Firstly, a separated-dough method was developed to increase structural heterogeneity. The gluten free crackers (F0) prepared by the method had lower hardness and showed voids and cavities in the structure. An increase in mixing time leads to heterogeneity reduction in structure and water distribution. Secondly, NC and FC were added into the formula as structuring materials and to partially replace flour and PGS for lower calorie content and higher fibre content. When NC was initially added to butter dough (F1, F3, and F6), the fibres were coated by lipid and less hydrated when mixed with water doughs and interactions with other hydrophilic materials were reduced. NC fibres were aligned during dough laminating and contributed to the formation of a fibrous and laminar structure in the end products as the skeleton of the crackers. Therefore, F1, F3 and F6 had higher thickness, hardness and fracturability; and sharper breakage and sound emission. These properties, especially high thickness and sharp sound emission, are usually observed for wheat crackers. When NC was added to the water dough (F2), the fibres were embedded in the matrix and extensively interacted with PSY, PGS and starch and proteins from rice flour. Therefore, it did not contribute to the formation of a laminar structure and had lower effects on hardness, fracturability and sound emission. However, the addition of NC in water dough inhibited the viscosity development of crackers during hydration which might be advantageous for a desirable mouthfeel. FC can be used to replace PGS at a lower addition level (F5) without noticeable influence on cracker structure. In addition, a reduction in viscosity during hydration was also observed.

\section{Conflicts of interest}

Bruce Linter is employed by PepsiCo, Inc. The views and opinions expressed in this manuscript are those of the authors and do not necessarily reflect the position or policy of PepsiCo, Inc.

\section{Acknowledgements}

This work was supported by the University of Nottingham (Vice-Chancellor's Scholarship for Research Excellence (International)) and PepsiCo, Inc. The authors would like to thank Khatija Nawaz Husain for her technical help with confocal laser scanning microscopy, which was conducted at the School of Life Sciences Imaging, and Microscopy Facility (SLIM) at the University of Nottingham. Scanning electron microscopic images were collected at the Nanoscale and Microscale Research Centre (nmRC) at the University of Nottingham.

\section{References}

1 I. Sedej, M. Sakač, A. Mandić, A. Mišan, M. Pestorić, O. Šimurina and J. Čanadanović-Brunet, Quality assessment of gluten-free crackers based on buckwheat flour, LWT-Food Sci. Technol., 2011, 44, 694-699.

2 O. Radočaj, E. Dimić and R. Tsao, Effects of hemp (Cannabis sativa L.) seed oil press-cake and decaffeinated green tea leaves (Camellia sinensis) on functional characteristics of gluten-free crackers, J. Food Sci., 2014, 79, C318C325.

3 S. A. Mir, S. J. D. Bosco, M. A. Shah, S. Santhalakshmy and M. M. Mir, Effect of apple pomace on quality characteristics of brown rice based cracker, J. Saudi Soc. Agric. Sci., 2017, 16, 25-32.

4 A. B. Martin-Diana, N. Izquierdo, I. Albertos, M. S. Sanchez, A. Herrero, M. A. Sanz and D. Rico, Valorization of carob's germ and seed peel as natural antioxidant ingredients in gluten-free crackers, J. Food Process. Preserv., 2017, 41, e12770.

5 N. Nammakuna, S. A. Barringer and P. Ratanatriwong, The effects of protein isolates and hydrocolloids complexes on dough rheology, physicochemical properties and qualities of gluten-free crackers, Food Sci. Nutr., 2016, 4, 143-155.

6 Y. Ren, B. R. Linter and T. J. Foster, Effects of psyllium seed husk powder, methylcellulose, pregelatinised starch, and cold water swelling starch on the production of gluten free crackers, Food Funct., 2021, DOI: 10.1039/D0FO03377D.

7 Y. Habibi, L. A. Lucia and O. J. Rojas, Cellulose nanocrystals: Chemistry, self-assembly, and applications, Chem. Rev., 2010, 110, 3479-3500.

8 A. F. Turbak, F. W. Snyder and K. R. Sandberg, Microfibrillated cellulose, US4374702A, 1983.

9 A. F. Turbak, F. W. Snyder and K. R. Sandberg, Food products containing microfibrillated cellulose, US4341807, 1982.

10 Y. Ren, B. R. Linter and T. J. Foster, Cellulose fibrillation and interaction with psyllium seed husk heteroxylan, Food Hydrocolloids, 2020, 104, 105725.

11 Y. Ren, B. R. Linter and T. J. Foster, Starch replacement in gluten free bread by cellulose and fibrillated cellulose, Food Hydrocolloids, 2020, 107, 105957.

12 International Fiber Corporation, Food applications SolkaFloc®, http://buyersguide.supplysideshow.com/media/54/ library/49964-313.pdf, (accessed October 2020).

13 S. Meiboom and D. Gill, Modified Spin-Echo method for measuring nuclear relaxation times, Rev. Sci. Instrum., 1958, 29, 688-691.

14 N. Sozer, L. Cicerelli, R.-L. Heiniö and K. Poutanen, Effect of wheat bran addition on in vitro starch digestibility, physico-mechanical and sensory properties of biscuits, J. Cereal Sci., 2014, 60, 105-113.

15 M. Ndife, G. Şumnu and L. Bayındırlı, Differential scanning calorimetry determination of gelatinization rates in different starches due to microwave heating, LWT-Food Sci. Technol., 1998, 31, 484-488. 
16 H. R. Tang, J. Godward and B. Hills, The distribution of water in native starch granules - a multinuclear NMR study, Carbohydr. Polym., 2000, 43, 375-387.

17 G. M. Bosmans, B. Lagrain, L. J. Deleu, E. Fierens, B. P. Hills and J. A. Delcour, Assignments of proton populations in dough and bread using nmr relaxometry of starch, gluten, and flour model systems, J. Agric. Food Chem., 2012, 60, 5461-5470.

18 M. Ritota, R. Gianferri, R. Bucci and E. Brosio, Proton NMR relaxation study of swelling and gelatinisation process in rice starch-water samples, Food Chem., 2008, 110, 14-22.

19 H. R. Tang, A. Brun and B. Hills, A proton NMR relaxation study of the gelatinisation and acid hydrolysis of native potato starch, Carbohydr. Polym., 2001, 46, 7-18.

20 M. R. Serial, M. S. Blanco Canalis, M. Carpinella, M. C. Valentinuzzi, A. E. León, P. D. Ribotta and R. H. Acosta, Influence of the incorporation of fibers in biscuit dough on proton mobility characterized by time domain NMR, Food Chem., 2016, 192, 950-957.

21 H. M. Wyss, K. Miyazaki, J. Mattsson, Z. B. Hu, D. R. Reichman and D. A. Weitz, Strain-rate frequency superposition: A rheological probe of structural relaxation in soft materials, Phys. Rev. Lett., 2007, 98, 238303.

22 M. Meerts, R. Cardinaels, F. Oosterlinck, C. M. Courtin and P. Moldenaers, The interplay between the main flour constituents in the rheological behaviour of wheat flour dough, Food Bioprocess Technol., 2017, 10, 249-265.

23 H. Mamat and S. E. Hill, Effect of ingredients on the mass loss, pasting properties and thermal profile of semi-sweet biscuit dough, J. Therm. Anal. Calorim., 2018, 134, 14131428.

24 C. G. Biliaderis, C. M. Page, T. J. Maurice and B. O. Juliano, Thermal characterization of rice starches: a polymeric approach to phase transitions of granular starch, J. Agric. Food Chem., 1986, 34, 6-14.

25 R. Kovrlija and C. Rondeau-Mouro, Hydrothermal changes of starch monitored by combined NMR and DSC methods, Food Bioprocess Technol., 2017, 10, 445-461.

26 J. W. Donovan, Phase transitions of the starch-water system, Biopolymers, 1979, 18, 263-275.

27 P. Díaz-Calderón, B. MacNaughtan, S. Hill, T. Foster, J. Enrione and J. Mitchell, Changes in gelatinisation and pasting properties of various starches (wheat, maize and waxy maize) by the addition of bacterial cellulose fibrils, Food Hydrocolloids, 2018, 80, 274-280.
28 K. Kohyama and K. Nishinari, Effect of soluble sugars on gelatinization and retrogradation of sweet-potato starch, J. Agric. Food Chem., 1991, 39, 1406-1410.

29 A. Zolelmein, S. Movahhed, M. H. Azizi and H. Ahmadi Chenarbon, Assessment of simultaneous addition of sucrose and xanthan effects on the thermal, pasting, and rheological behavior of corn starch, J. Texture Stud., 2020, 51, 453-463.

30 M. D. Torres, R. Moreira, F. Chenlo and M. H. Morel, Effect of water and guar gum content on thermal properties of chestnut flour and its starch, Food Hydrocolloids, 2013, 33, 192-198.

31 M. Kaur, K. S. Sandhu, A. Arora and A. Sharma, Gluten free biscuits prepared from buckwheat flour by incorporation of various gums: Physicochemical and sensory properties, LWT-Food Sci. Technol., 2015, 62, 628-632.

32 E. A. Foegeding, C. J. Vinyard, G. Essick, S. Guest and C. Campbell, Transforming structural breakdown into sensory perception of texture, J. Texture Stud., 2015, 46, 152-170.

33 G. Roudaut, C. Dacremont, B. Vallès Pàmies, B. Colas and M. Le Meste, Crispness: a critical review on sensory and material science approaches, Trends Food Sci. Technol., 2002, 13, 217-227.

34 Z. M. Vickers, Crackliness: Relationships of auditory judgments to tactile judgments and instrumental acoustical measurements, J. Texture Stud., 1984, 15, 49-58.

35 A. Zdunek, D. Konopacka and K. Jesionkowska, Crispness and crunchiness judgment of apples based on contact acoustic emission, J. Texture Stud., 2010, 41, 75-91.

36 E. Çarşanba, K. Duerrschmid and G. Schleining, Assessment of acoustic-mechanical measurements for crispness of wafer products, J. Food Eng., 2018, 229, 93101.

37 J. Chen, C. Karlsson and M. Povey, Acoustic envelope detector for crispness assessment of biscuits, J. Texture Stud., 2005, 36, 139-156.

38 P. Varela, A. Salvador and S. M. Fiszman, Methodological developments in crispness assessment: Effects of cooking method on the crispness of crusted foods, LWT-Food Sci. Technol., 2008, 41, 1252-1259.

39 D. Manley, Classification based on enrichment of the formulation, in Technology of biscuits, crackers and cookies, ed. D. Manley, Woodhead Publishing, Cambridge, 3rd edn, 2000. 